\title{
Modelling Elasticity in Solids using Active Cubes - Application to Simulated Operations
}

\author{
Morten Bro-Nielsen ${ }^{1}$ \\ Institute of Mathematical Modelling \\ Technical University of Denmark, Bldg. 321 \\ DK-2800, Lyngby, Denmark \\ e-mail: bro@imm.dtu.dk
}

November 14, 1994

\begin{abstract}
This paper describes an approach to elastic modelling of human tissue based on the use of 3D solid active models - active cubes [2] - and a shape description based on the metric tensor in a solid. Active cubes are used because they provide a natural parameterization of the surface and the interior of the given object when deformed to match the object's shape. Using the metric tensor to store the shape of the deformed active cube the elastic behaviour of the object in response to applied forces or subject to constraints is modelled by minimizing an energy based on the metric tensor. The application of this approach to modelling the elastic deformation of human tissue in response to movement of bones is demonstrated.

Categories: Virtual patients for surgical training, building and using physical deformable models.
\end{abstract}

\section{Introduction}

The purpose of simulated operations is to enable a surgeon to experiment with different surgical procedures in an artificial environment. Applications include the training of surgeons and for practice before difficult operations.

Generally, simulated operations may be separated into two distinct but overlapping processes: visualization of the actual surgical procedure using artificial scapels etc.; and modelling the results of operations. The latter is used in particular for determining the results of craniofacial surgery to remove craniofacial

\footnotetext{
${ }^{1}$ Currently with the INRIA Epidaure group, 2004, route des Lucioles, 06902 Sophia Antipolis cedex, France
} 
deformations. The purpose of a simulated operation system in this case is to evaluate and visualize the results of operations on the overall facial shape of the patient. Although relatively few craniofacial operations are performed each year, their impact can be profound; in some cases changing the life of an individual. Simulation of the results is not only important for the surgeon, because such operations are difficult, but also to demonstrate to the patient and relatives the potential results of the often painful surgery and the long, slow recovery

Previously, simulated operations have been performed using surface models. For example, Caponetti et.al. [3] used a surface model reconstructed using the occluding contours in two x-rays to simulate bone surgery. Surgical reconstruction of the skull, where the skull is cut into several pieces and subsequently assembled into a new shape, has been modelled by several teams $[6,10,11,15]$. They all used surface models, although Delingette et.al. [6] connected the surface of the bones and the surface of the skin with 'muscles' defined as a 3D structure, thereby obtaining a pseudo-3D model. In some cases, the influence of bone movement on facial features has been modelled. Yasuda et.al. [15] used an approximate method to put soft tissue onto the skull after surgery, based on the thickness of the soft tissue before the operation. Delingette et.al. [6] used the 'muscles' referred to above to model the deformation of the face. In both cases, the approach only supported a restricted set of operations, relevant to their application, and in the latter involved operator intervention for application of 'muscles'.

The approach proposed here involves complete 3D modelling of the solid - as opposed to surface-structure of the object. It is quite general, and is for example able to model elastic deformation of soft tissue in response to applied forces and constraints such as moved bones. An example of a simulated operation involving movement of the jaw is used to illustrate the results.

An extension of the Snake family of active models called active cubes [2] is used to model the shape of the given object. A $2 \mathrm{D}$ version called active nets was proposed by Tsumiyama, Sakaue et.al. [9, 13]. Active cubes parameterize a 3D solid shape and differ from previously proposed active models [8,5] by having nodes in the interior of the object they model. It follows that the interior of an active cube is defined, unlike in the case of surface models. Performing simulated operations, that include cutting into the model, is straightforward with active cubes because of the interior nodes. Intuitively, a cut corresponds to deleting the connection between two nodes on the surface of the active cube. For a surface model, a cut creates a hole in the model; with an active cube, the revealed interior nodes become new boundary nodes that model the interior bared by the cut.

Interior nodes also make active cubes suitable for modelling the $3 \mathrm{D}$ elastic behaviour of solids. Because the parameterization of the active cube is inherent in its structure, deforming an active cube to match a given object, provides a simple discrete parameterization of the object in curvilinear coordinates. After deformation of the active cube to match the $3 \mathrm{D}$ object, the equilibrium shape is stored in the active cube using the metric tensor $[7,12]$. The metric tensor stores 
distances and angles and is therefore useful as a measure of shape. It has been used previously for shape description in $[1,12]$. Elastic deformation of the head is modelled using an energy measure based on the metric tensor. This energy measures the accumulated deviation in terms of distance and angles of the local deformation. When constraints or forces are applied to the active cube, energy minimization techniques can be used to determine an equlibrium shape which is a compromise between the original shape of the object and the constraints applied to it. This is used in practice to simulate the elastic deformation of soft tissue due to movement of included bones.

\section{Modelling Object Shape with Active Cubes}

The data used in this context are usually 3D MR or CT scans of human body parts providing a $3 \mathrm{D}$ voxel data set. Although elastic modelling could be applied to voxel data, the amount of processing would be prohibitive. It is, therefore, necessary to reduce the resolution of the data. This is accomplished by matching a deformable $3 \mathrm{D}$ model to the data using a discretization which has a resolution orders of magnitude less than the original data set.

To model the 3D data set, a 3D active cube [2] is used. An active cube is a parameterization of a subspace of the data space $\mathcal{R}^{3}$ defined by $\boldsymbol{v}(r, s, t)=$ $(x(r, s, t), y(r, s, t), z(r, s, t))$, where $(r, s, t) \epsilon\left([0,1]^{3}\right)$. By discretizing this subspace, a 3D mesh of nodes is defined in the subspace defined by node $e_{i j k}=$ $(i l, j m, k n)$ where $(l, k, m)$ are the discretization steps used.

To control the deformation of the active cube an energy function is defined by:

$$
E(\boldsymbol{v})=\int_{0}^{1} \int_{0}^{1} \int_{0}^{1} E_{\text {int }}(\boldsymbol{v}(r, s, t))+E_{e x t}(\boldsymbol{v}(r, s, t)) d r d s d t
$$

where $E_{\text {int }}$ is the internal energy of the net, that controls the shape and structure of the net and $E_{\text {ext }}$ is the energy due to external and image forces.

The role of the internal energy term is to preserve the rigid structure of the node mesh, to smooth irregularities on the surface, and to force the active cube to contract. The internal energy can be defined straightforwardly using first and second order derivatives:

$$
\begin{aligned}
E_{i n t}(\boldsymbol{v}(r, s, t)) & \left.=\left(\alpha\left(\left|\boldsymbol{v}_{r}(r, s, t)\right|^{2}+\left|\boldsymbol{v}_{s}(r, s, t)\right|^{2}\right)+\left|\boldsymbol{v}_{t}(r, s, t)\right|^{2}\right)\right) \\
& +\left(\beta \left(\left|\boldsymbol{v}_{r r}(r, s, t)\right|^{2}+\left|\boldsymbol{v}_{s s}(r, s, t)\right|^{2}+\left|\boldsymbol{v}_{t t}(r, s, t)\right|^{2}\right.\right. \\
& \left.+\left(\gamma\left(2\left|\boldsymbol{v}_{r s}(r, s, t)\right|^{2}+2\left|\boldsymbol{v}_{r t}(r, s, t)\right|^{2}+2\left|\boldsymbol{v}_{s t}(r, s, t)\right|^{2}\right)\right)\right)
\end{aligned}
$$

where subscripts signifies partial derivatives and $\alpha, \beta$ and $\gamma$ are coefficients controlling the first and second order smoothness of the net. The internal energy has the same form and functionality as that used with other active models of the Snake family $[8,5]$ : the first derivatives control contraction, and the second derivatives control bending and twisting. 
The external energy is designed to attract the active cube to image objects which, in this context, are assumed to have high image intensities. Because of the solid structure of the active cube, the external energy is not defined using edge data which is usually used for surface/contour models. Instead the actual image data is used. The general form is:

$$
\begin{aligned}
E_{\text {ext }}(\boldsymbol{v}(r, s, t))= & \omega f[I(\boldsymbol{v}(r, s, t))]+ \\
& \frac{\rho}{|\mathcal{N}(r, s, t)|} \sum_{\boldsymbol{p}_{\epsilon \mathcal{N}(r, s, t)} \frac{1}{\|\boldsymbol{v}(r, s, t)-\boldsymbol{v}(\boldsymbol{p})\|} f[I(\boldsymbol{v}(\boldsymbol{p}))]} \\
f[I(\boldsymbol{v}(r, s, t))]= & \begin{cases}h\left[I_{\text {max }}-\overline{I(\boldsymbol{v}(r, s, t))_{n}}\right] & \text { for internal nodes } \\
h[I(\boldsymbol{v}(r, s, t))] & \text { for boundary nodes }\end{cases}
\end{aligned}
$$

where $\omega$ and $\rho$ are weights, $\mathcal{N}(r, s, t)$ is the neighbourhood of the node $(r, s, t)$ not including the node itself, $\overline{I(\boldsymbol{v}(\boldsymbol{p}))_{n}}$ is the mean intensity in a $n \times n \times n$ cube, $I_{\max }$ is the maximum image intensity and $h$ is an appropriate scaling function.

This energy is basically a sum of two terms: the first is a function of the image intensity at the current node; the other is the weighted contributions from the neighbouring nodes. $f$ is defined differently for internal and boundary nodes because of the different roles assigned to these two groups of nodes. Internal nodes are attracted to volumes with high intensities corresponding to image objects, whereas boundary nodes are repulsed from the same volumes. In practice, the boundary nodes limit the contraction of the active cube which is caused by the internal energy and the internal nodes.

Energy minimization is performed locally using an improved version of the finite difference Greedy algorithm $[2,14]$.

Unfortunately, the number of nodes in an active cube is very high, compared to other active models, because of the internal nodes. The computing time is, therefore, not irrelevant. In practice, it can be reduced by fixing the $z$-component of $\boldsymbol{v}=(x, y, z)$. In this case, nodes are only allowed to move inside a slice; not between slices. This typically reduces the computing time by half.

\section{Shape Quantification}

In order to model the elastic deformation of the active cube with a given shape as a reference, a quantification of the shape must be determined. A practical quantification measure is the metric tensor, or the first fundamental form, of a solid [1, 12], which is a 3 by 3 matrix defined by:

$$
G_{i j}(\boldsymbol{v}(\boldsymbol{p}))=\frac{\partial \boldsymbol{v}}{\partial x_{i}} \cdot \frac{\partial \boldsymbol{v}}{\partial x_{j}}
$$

where $x_{i}$ is the $i$ 'th element of $(x, y, z)$. The metric tensor stores the shape of a solid defined by distances and angles. 
The parameterization of the active cube is a manifold with codimension 0 , meaning that the dimension of the parameter and image space is the same. The shape of a manifold with codimension $c$ is described by $c$ curvature tensors and the metric tensor $[7,12]$. Because the codimension is zero, only the metric tensor is needed.

The metric tensor is known in elasticity theory as the Right Cauchy-Green tensor. It is a general theorem [4] that this tensor is invariant to rigid transformations (rotation, transformation). It also follows, that two shapes which can be obtained from one another with a rigid transformation, will correspond to the same tensor.

\section{Elastic Deformation}

In order to handle elastic deformations of the active cube from a reference object shape, the following energy measure is defined to quantify the shape deviation from the reference shape $\boldsymbol{G}^{o}[12]$ :

$$
E_{\text {Shape }}(\boldsymbol{v})=\int_{0}^{1} \int_{0}^{1} \int_{0}^{1} \sum_{i, j=1}^{3} w_{i j}\left(G_{i j}-G_{i j}^{o}\right)^{2} d r d s d t
$$

where $w_{i j}$ are weights determining the influence of the different tensor components. For an isotropic material, there is only two weights, corresponding to off- and on-diagonal elements of the metric tensor. The off-diagonal elements quantify angle deviations and the diagonal elements distance deviations.

When constraints or forces are applied to the active cube, the equilibrium shape of the active cube is determined by minimizing the shape energy $E_{\text {Shape }}$ under the given constraints.

\section{$5 \quad$ Simulating Operations}

This section applies the framework presented above to model the movement of bone structures in a human body. First, an artificial example is used to illustrate the procedure; then the movement of the jaw in a CT scan is shown.

The active cube model is used to model the shape of the object, and the metric tensor is used to quantify the reference shape and changes to this. The general procedure is the following:

1. Deform an active cube to model the object.

The active cube is initialized around the object and allowed to contract to model it. The result is a discrete parameterization in curvilinear coordinates of the object. 
2. Determine the equilibrium shape using the metric tensor.

The metric tensor is determined and stored at all nodes in the active cube.

3. Fix the position of nodes in the active cube which are positioned in image voxel labelled as bone.

It is assumed that bones have been extracted from the image. In CT scans, this is straightforward.

Fixing the position, means that subsequent elastic deformation will not be applied to these nodes. This way, only the soft, non-bone, tissue will move elastically.

4. Determine the bones to be moved. Move the corresponding active cube nodes to the required new position.

5. Using the final position of the bone nodes as constraints, minimize the $E_{\text {Shape }}$ energy.

The equilibrium position is the resulting shape deformation.

In some cases, large movements of bones can cause problems if they are larger than the discretization steps in the cube. In this case, making one big movement causes connections in the cube to overlap which can lead to spurious results. A solution to this problem is to determine a path to the desired new position of the bone nodes, and then move the bone nodes in steps. After each step movement, energy minimization is performed reaching an intermediary shape.

\subsection{Experimental Results}

First results with an artificial example illustrates the simulated operation algorithm. The example is supposed to illustrate an orthogonal slice of a leg. In figure 1, a drawing of the scene is shown. The operation consists of moving a piece of bone outwards from its reference position.

First, an active cube was allowed to deform to match the reference shape of the test data set. A 3D rendering of the active cube after the first initial movement of the bone is shown in figure 2. Because the elastic energy minimization has not been applied yet the soft tissue is just moved outwards, corresponding exactly to the movement of the bone.

Figure 3 shows the result after elastic deformation has been modelled using energy minimization under the constraints posed by the bones. This clearly demonstrates the elastic behaviour of the soft tissue in response to the bone movement.

Figure 4 shows an active cube after having contracted around a CT scan of a head. The 'scar' on the head is due to a shading problem occurring at the

corner of the cube. The surface normal is not properly smoothed here by the 


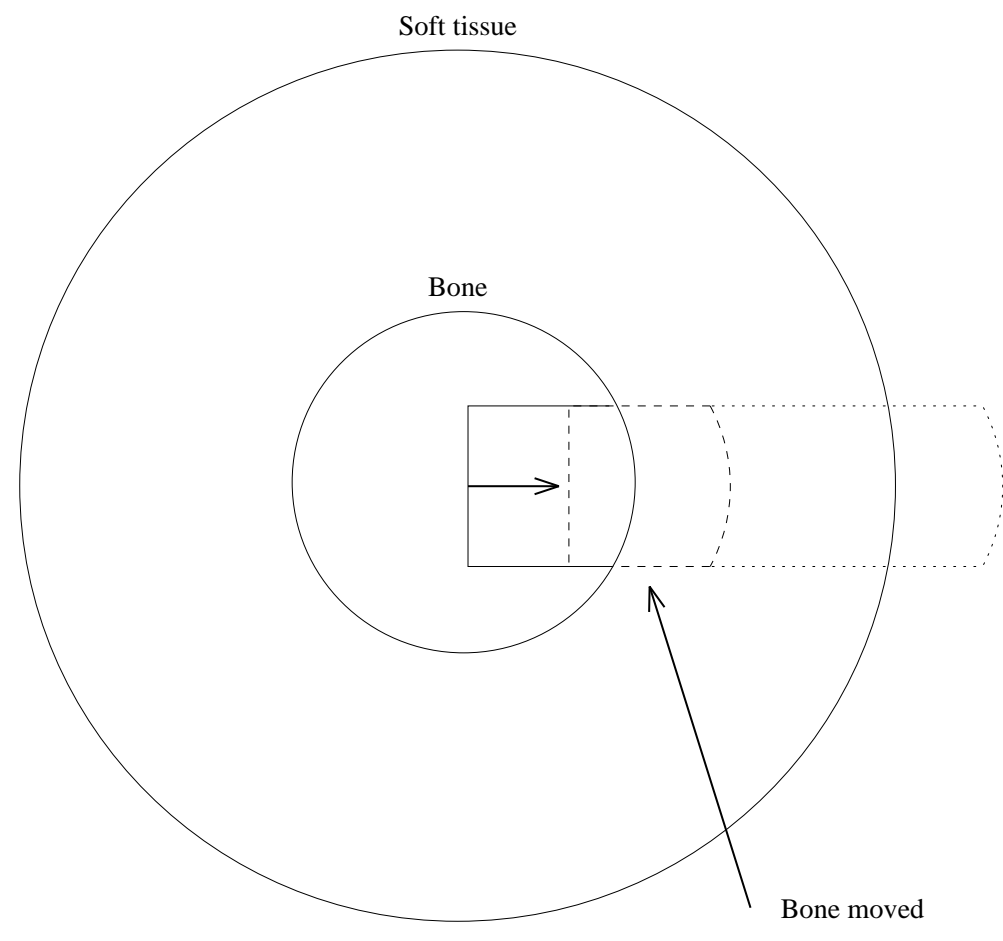

Figure 1. Test object for simulated operation.

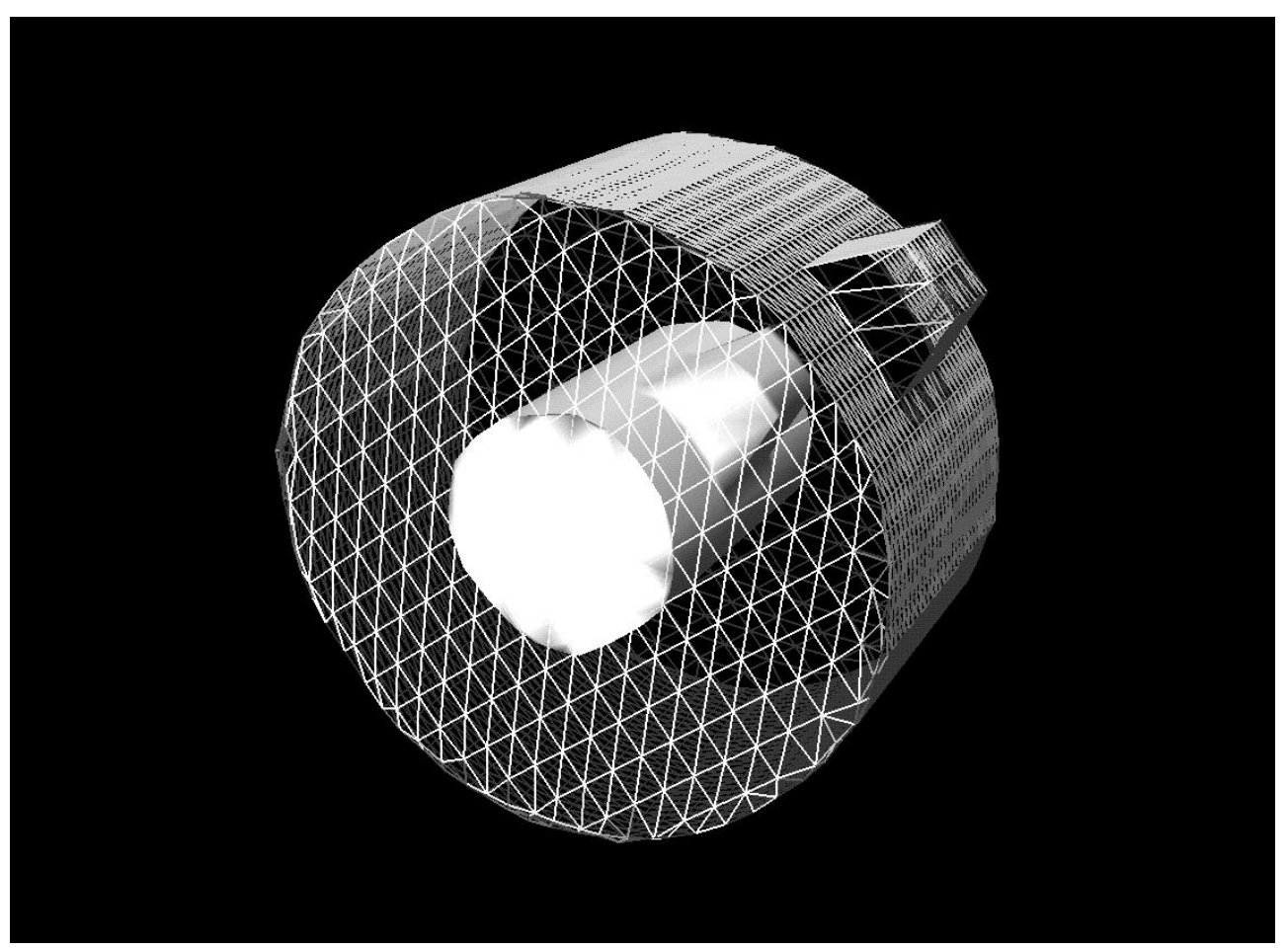

Figure 2. The active cube after initial movement modelling the test object and movement of hard tissue. 

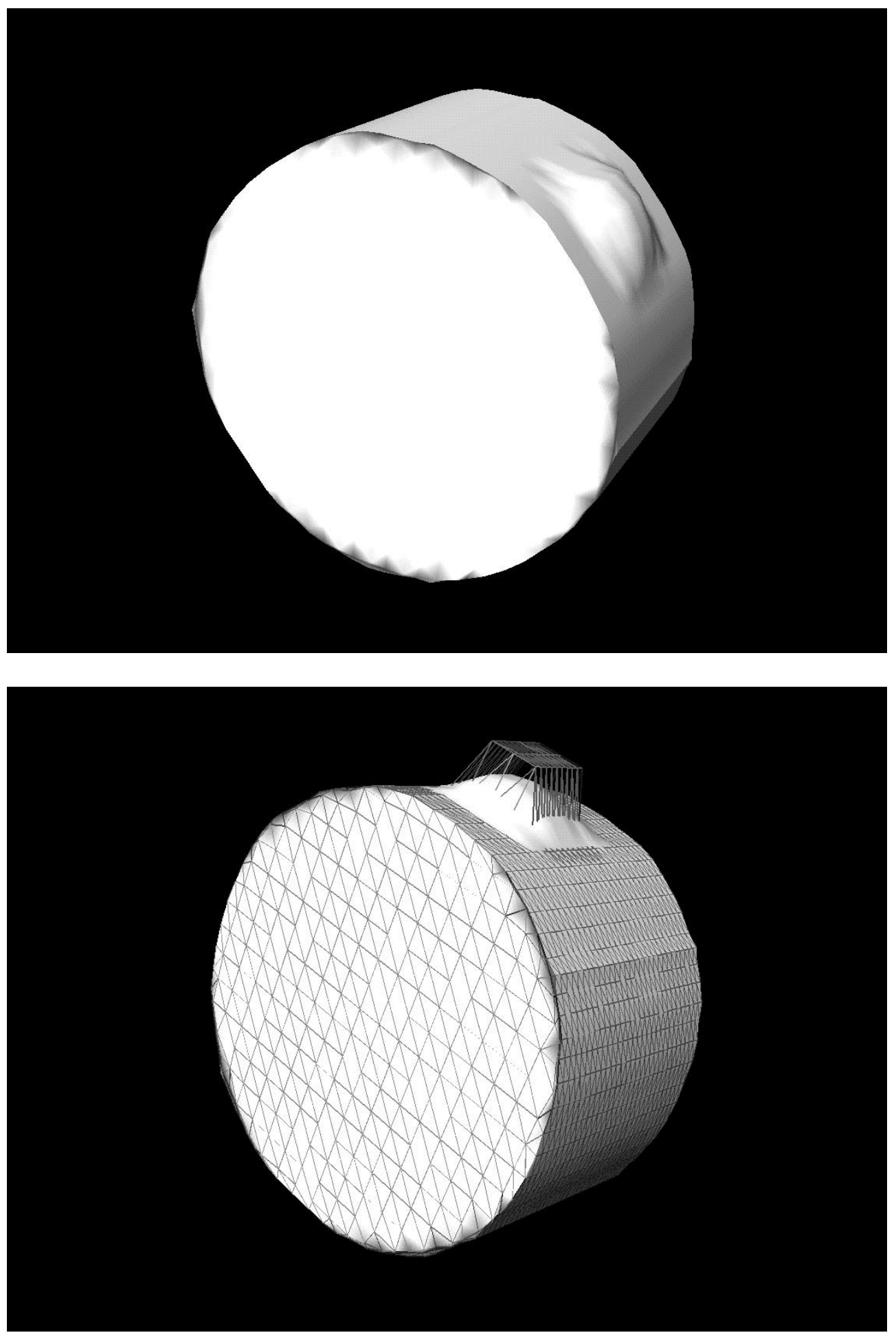

Figure 3. The resulting active cube after the elastic behaviour of the soft tissue has been simulated. Bottom: With the initial movement overlaid as a net. 


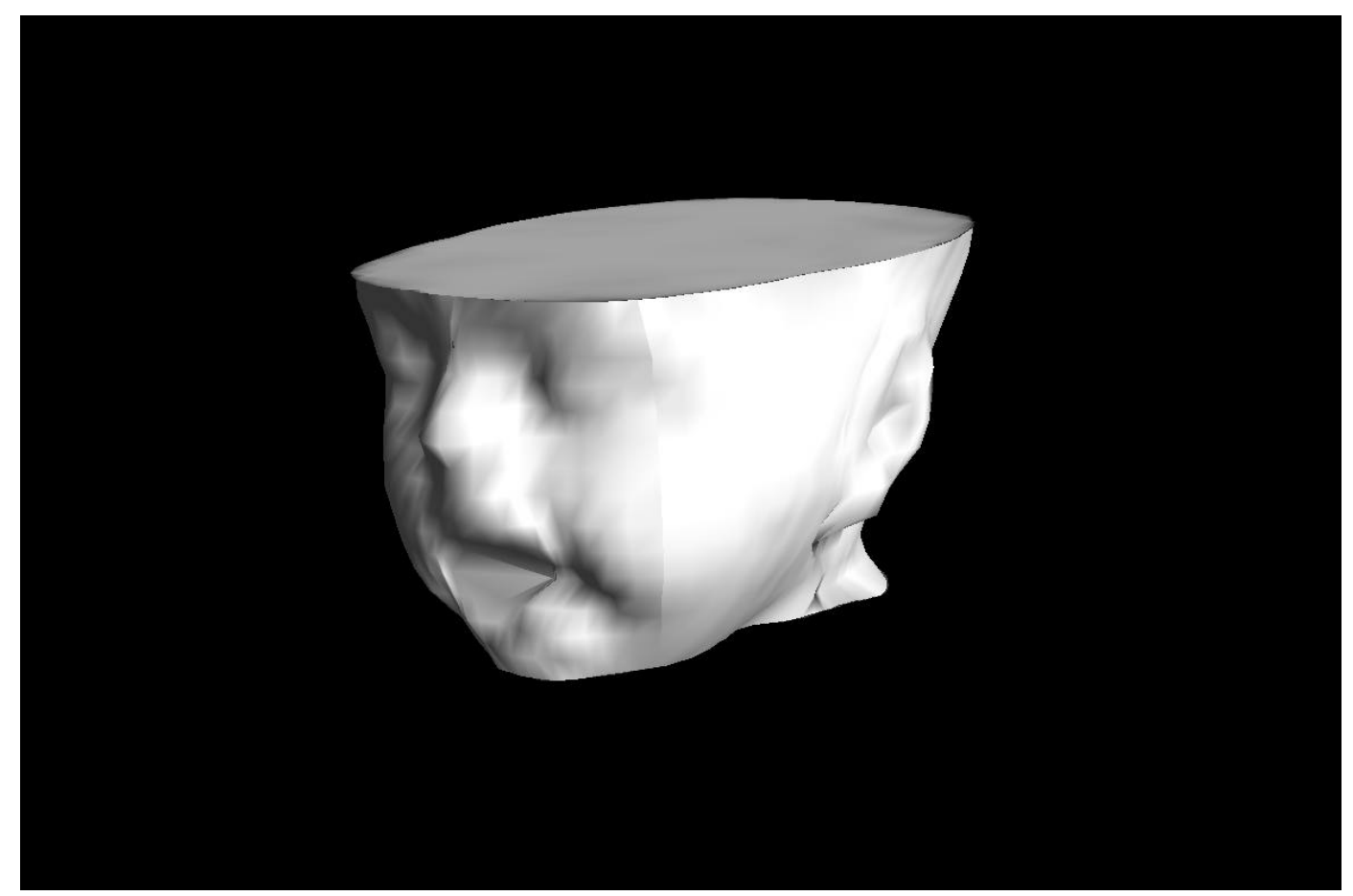

Figure 4. The result after applying the 3D active cube to the CT scan of a head.

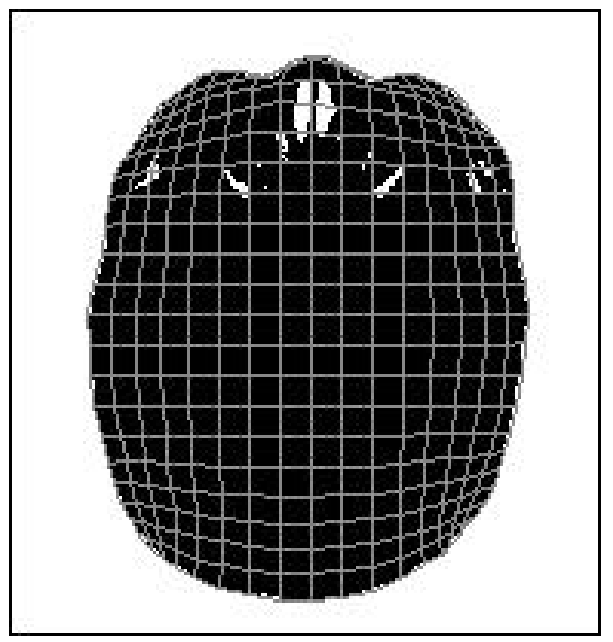

Figure 5. The interior of the $3 \mathrm{D}$ active cube. 


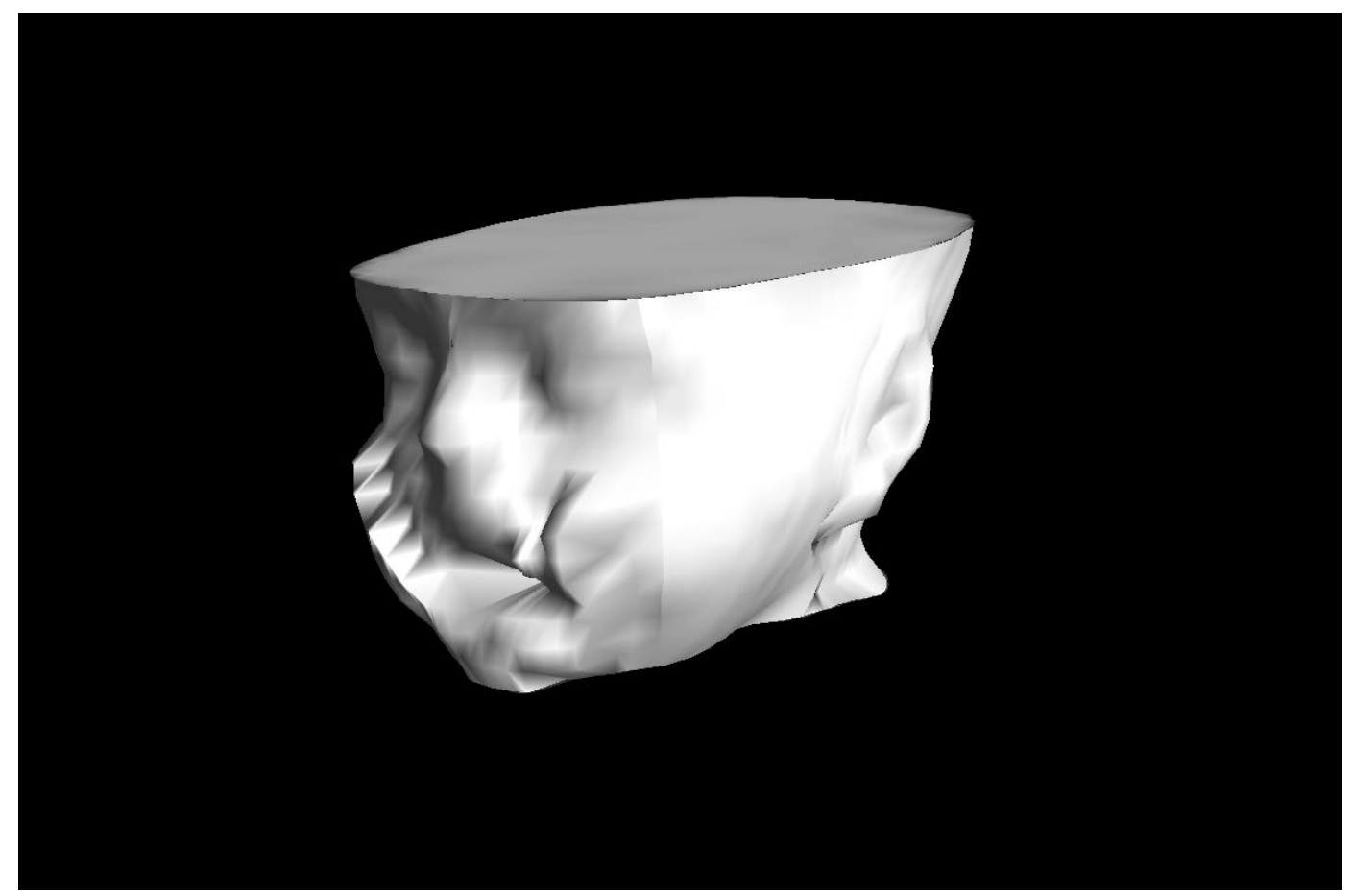

Figure 6. Active cube after the initial movement of the jaw.

software used for rendering the surface. The deformation of the right side of the mouth is caused by a tube that was inserted into the patient's mouth during image aquisition.

Figure 5 shows the interior of the resulting $3 \mathrm{D}$ active net. The regular structure of the nodes in the interior is clearly perceived. Notice, that nodes have not moved between slices, because the $z$-component were fixed during this experiment. To simulate an operation, the jaw was moved approximately $1 \mathrm{~cm}$ outwards horizontally. Figure 6 shows the active cube after the initial movement corresponding to figure 2. Figure 7 shows the result after energy minimization. In figure 7 /bottom it is possible to see how the movement has influenced the soft tissue. Notice the inward contraction of the soft tissue on the chin because of the elongation of the face.

\section{Conclusion}

A approach to modelling elastic deformations in 3D solid objects has been proposed. Using the active cube introduced in [2], the solid 3D structure has been modelled, providing a curvilinear, discrete parameterization of the object.

The active cube subsequently provided the basis for quantizing the shape of the object using the metric tensor. Using an energy measure based on the metric tensor, elastic deformations have been modelled successfully. 

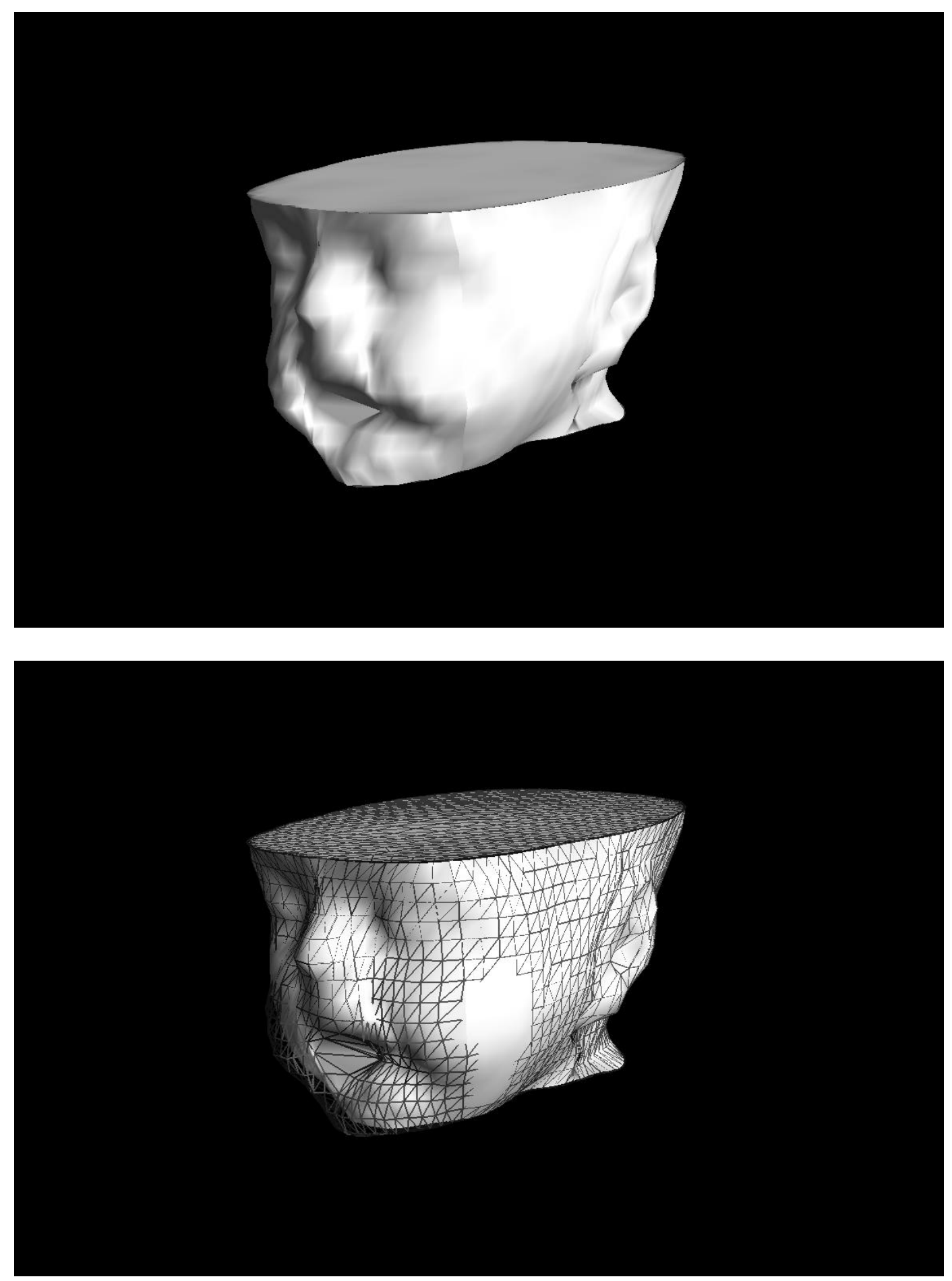

Figure 7. Active cube after elastic deformation under constraints of jaw movements. 
Simulation of an operation involving movement of bone in a human head allows the user to perceive the elastic deformation of the soft tissue of the patient's head.

In general we believe that using 3D solid models for operation simulation and 3D solid shape measurement has considerable promise. Simulated operations involving movement of bone have been demonstrated here. However it is obvious that simulated operations that include cutting into the pseudo-patient also require $3 \mathrm{D}$ solid models to enable visualization both of the interior of the patient and the actual elastic behaviour of the patient's soft tissue during cutting.

Using 3D solid active cubes increases the computational complexity of the algorithms; but the time required is not prohibitive. In the case of modelling the head in figure 4, the deformation of the active cube to match the head took 15 minutes on a SGI Indigo II using an inefficient program. Modelling the elastic deformation of the soft tissue after movement of the jaw was performed in less than 1 minute. In practice, extracting the jaw was the most time-comsuming process.

\section{Acknowledgement}

The CT scan was supplied by Dr. Sven Kreiborg of the Dentist School of Copenhagen.

\section{References}

[1] K.A. Bartels, A.C. Bovik and C.E. Griffin: Spatio-temporal tracking of material shape change via multi-dimensional splines, Proc. IEEE Workshop Biomedical Image Analysis, Seattle, USA, pp. 110-116, June 1994

[2] M. Bro-Nielsen: Active nets and cubes, submitted to CVGIP: Image Understanding, 1994

[3] L. Caponetti and A.M. Fanelli: Computer-aided simulation for bone surgery, IEEE Computer Graphics \& Applications, November, pp. 86-92, 1993

[4] P. G. Ciarlet: Mathematical elasticity, vol. I: Three-dimensional elasticity, North-Holland, ISBN 0-444-70259-8 (v. 1), 1988

[5] L. D. Cohen and I. Cohen: Finite-element methods for active contour models and balloons for 2-D and 3-D images, IEEE Trans. Pattern Analysis and Machine Intelligence, vol. 15, no. 11, pp. 1131-1147, 1989

[6] H. Delingette, G. Subsol, S. Cotin and J. Pignon: A craniofacial surgery simulation testbed, INRIA Tech. Rep. 2199, 1994 
[7] M.P. Do Carmo: Differential geometry of curves and surfaces, USA, Prentice-Hall, 1976

[8] M. Kass, A. Witkin and D. Terzopoulos: Snakes: Active contour models, Int. J. of Computer Vision, vol. 2, pp. 321-331, 1988

[9] K. Sakaue and K. Yamamoto: Active net model and its application to region extraction, JITE of Japan, vol. 45, no. 10, pp. 1155-1163, 1991

[10] J. Satoh, H. Ciyokura, M. Kobayashi and T. Fujino: Simulation of surgical operations based on solid modelling, in T. L. Kunii editor: Visual computing, Integrating computer graphics with computer vision, Tokyo (Japan), Computer Graphics Society, Springer Verlag, pp. 907-916, 1992

[11] R.H. Taylor: An overview of computer assisted surgery research at IBM T.J. Watson research center, in Innovation et technologie en biologie et medecine, 1992

[12] D. Terzopoulos: Deformable models, The Visual Computer, vol. 4, pp. 306331,1988

[13] Y. Tsumiyama, K. Sakaue and K. Yamamoto: Active net: Active net model for region extraction (in Japanese), IPSJ SIG Notes, CV 63-2, vol. 89, no. 96, pp. 1-8, 1989

[14] D. J. Williams and M. Shah: A fast algorithm for active contours and curvature estimation, CVGIP: Image Understanding, vol. 55, no. 1, pp. 14-26, 1992

[15] T. Yasuda, Y. Hashimoto, S. Yokoi and J-I. Toriwaki: Computer system for craniofacial surgical planning based on CT images, IEEE Trans. Medical Imaging, vol. 9, no. 3, pp. 270-280, 1990 